Published in final edited form as:

Nat Chem Biol. 2013 April ; 9(4): 220-221. doi:10.1038/nchembio.1208.

\title{
Put a ring on it
}

\author{
Amy C. Rosenzweig \\ Department of Molecular Biosciences and the Department of Chemistry, Northwestern University, \\ Evanston, Illinois, USA \\ Amy C. Rosenzweig: amyr@northwestern.edu
}

\begin{abstract}
The structure of a bacterial methane oxidation complex reveals how protein-protein interactions can mediate coupling of proton, electron and substrate delivery to a metal active site.
\end{abstract}

Nature's methane oxidation catalyst is methane monooxygenase (MMO), the primary metabolic enzyme in bacteria that subsist on methane gas ${ }^{1}$. MMOs are metalloenzymes that use $\mathrm{O}_{2}$ to convert methane to liquid methanol, a chemically difficult reaction that requires breaking the strong methane $\mathrm{C}-\mathrm{H}$ bond $\left(104.9 \mathrm{kcal} \mathrm{mol}^{-1}\right)$. There are two forms of MMO, a membrane-bound enzyme that uses copper $(\mathrm{pMMO})^{2}$ and a soluble enzyme that uses iron $(\mathrm{sMMO})^{3}$. The sMMO system includes a hydroxylase $(\mathrm{MMOH})$ that houses the catalytic diiron center, a reductase (MMOR) that supplies reducing equivalents to the active site and a 'coupling' or regulatory protein (MMOB) that is required to make the chemistry happen. Although the essential role of MMOB has been known for almost 30 years ${ }^{4}$, the molecular basis for its requirement has remained elusive despite the availability of $\mathrm{MMOH}^{5}$ and $\mathrm{MMOB}^{6}$ structures. In a tour de force 20 years in the making, Lee et al. ${ }^{7}$ now solve this riddle with the crystal structure of the $\mathrm{MMOH}-\mathrm{MMOB}$ protein-protein complex.

Understanding MMO enzymes is increasingly important in the quest to use abundant and relatively cheap natural gas supplies as fuel and chemical feedstocks. MMOs also provide a potential strategy for mitigating the effects of methane on global warming, and sMMO has been targeted for bioremediation applications because it oxidizes a myriad of other substrates besides methane ${ }^{1}$. However, it has been difficult to manipulate sMMO for these purposes without fully understanding the fundamental mechanisms controlling activity. The sMMO diiron active site is housed within a four-helix bundle in the a subunits of an $\alpha_{2} \beta_{2} \gamma_{2}$ polypeptide arrangement ${ }^{5}$. In the diiron(iii) oxidation state, the two irons are coordinated by two histidines and four glutamic acid residues as well as two bridging hydroxides. Upon reduction to the diiron(ii) state by MMOR, the solvent ligands disappear, and one of the glutamic acid ligands, Glu243, adopts a different coordination mode. This diiron(ii) center reacts with $\mathrm{O}_{2}$ to form a peroxodiiron(iii) intermediate that is converted to intermediate $\mathrm{Q}$, a diiron(iv) species responsible for methane oxidation. At some point during this cycle, protons are also transferred to the active site $^{3}$. These steps require the presence of MMOB,

(c) 2013 Nature America, Inc. All rights reserved.

Competing financial interests

The author declares no competing financial interests. 
which is coregulated with the three $\mathrm{MMOH}$ subunits ${ }^{1}$ and is known to affect the redox potential of the diiron site as well as the rate and regioselectivity of substrate oxidation ${ }^{8}$.

How MMOB facilitates these events has finally become clear in the $2.9-\AA$ resolution structure of the MMOH-MMOB complex from Methylococcus capsulatus (Bath) ${ }^{7}$. Successful crystallization of the protein-protein complex was most likely due to refinement of the MMOH purification procedure, which differs from that used many years ago to obtain the original MMOH samples for crystallization'. MMOB occupies the 'canyon' region ${ }^{5}$ on the MMOH surface, similar to what is observed for the related enzymes phenol hydroxylase ${ }^{9}$ and toluene-4-monooxygenase ${ }^{10}$ in complex with their regulatory proteins. However, unlike these other systems, MMOB has an extended $\mathrm{N}$ terminus that folds upon interaction with $\mathrm{MMOH}$, forming an unusual ring-like structure (Fig. 1) mediated by interprotein hydrogen bonds and hydrophobic interactions. This N-terminal region is essential for enzyme activity and anchors MMOB in place near the iron-coordinating fourhelix bundle, setting up a direct line of communication from MMOB to MMOH residues in the first and second coordination spheres of the diiron center. Several MMOH residues long hypothesized to have important roles in catalysis are affected, including Thr213 and Phe188. An MMOB-induced rotation of Thr213 leads to new hydrogen bonds with MMOH Glu240, which shifts inward toward the active site, closing a solvent-accessible pore proposed to have a role in proton transfer (Fig. 1). Thus, binding of MMOB may control delivery of the two protons needed to form the peroxodiiron(iii) and Q intermediates, perhaps mediated by MMOH Glu240. As this first door to the active site closes, another one opens. Binding of MMOB also alters a network of hydrophobic cavities proposed to provide methane and $\mathrm{O}_{2}$ access to the catalytic center ${ }^{3}$. In structures of $\mathrm{MMOH}$ alone, the active site cavity is isolated from this access route by residues Leu110 and Phe188. Upon binding MMOB, Phe188 shifts conformation, opening the gate and allowing the two small gaseous substrates access to the diiron center (Fig. 1). These results suggest that MMOB controls proton delivery and substrate access simultaneously. Finally, MMOB affects the diiron site itself, inducing a shift in coordination geometry of ligand Glu243. The new coordination is similar to that observed in reduced $\mathrm{MMOH}$. This change provides an explanation for previously observed perturbations of the active site structure by $\mathrm{MMOB}^{8}$ and suggests a role for Glu243 in proton transfer.

The MMOH-MMOB structure reveals how sMMO can use protein-protein interactions to link proton-coupled electron transfer with methane oxidation. The structural details explain a multitude of previous observations and also suggest new lines of investigation. The proposed roles of specific $\mathrm{MMOH}$ residues affected by MMOB binding may be further elucidated by site-directed mutagenesis, although it remains difficult to obtain large quantities of MMOH variants. The availability of MMOH-MMOB crystals also presents the tantalizing possibility that structural information on reactive intermediates may be within grasp. For the broader family of bacterial multicomponent monooxygenases ${ }^{8}$, the differences between MMOB and the other regulatory proteins, particularly in the N-terminal region, warrant further investigation. Also of interest is whether pMMO similarly regulates substrate access and proton transfer, using components that are yet to be identified. It may be that the pMMO transmembrane region provides an alternative means of coupling. 
Crystallization of protein-protein complexes is often difficult, but this accomplishment suggests that the brute force approach-persistence with biochemistry and crystallizationcan pay off in the long run. The MMOH-MMOB structure beautifully demonstrates how the architecture of a large enzyme complex precisely controls its chemistry. This control is one reason why MMOs are so selective for methane and presents a daunting challenge for developing biomimetic catalysts.

\section{References}

1. Jiang H, et al. Biochem Eng J. 2010; 49:277-288.

2. Culpepper MA, Rosenzweig AC. Crit Rev Biochem Mol Biol. 2012; 47:483-492. [PubMed: 22725967]

3. Tinberg CE, Lippard SJ. Acc Chem Res. 2011; 44:280-288. [PubMed: 21391602]

4. Colby J, Dalton H. Biochem J. 1978; 171:461-468. [PubMed: 418777]

5. Rosenzweig AC, Frederick CA, Lippard SJ, Nordlund P. Nature. 1993; 366:537-543. [PubMed: 8255292]

6. Walters KJ, Gassner GT, Lippard SJ, Wagner G. Proc Natl Acad Sci USA. 1999; 96:7877-7882. [PubMed: 10393915]

7. Lee SJ, McCormick MS, Lippard SJ, Cho US. Nature. 2013; 494:380-384. [PubMed: 23395959]

8. Wallar BJ, Lipscomb JD. Chem Rev. 1996; 96:2625-2658. [PubMed: 11848839]

9. Sazinsky MH, Dunten PW, McCormick MS, DiDonato A, Lippard SJ. Biochemistry. 2006; 45:15392-15404. [PubMed: 17176061]

10. Bailey LJ, McCoy JG, Phillips GN, Fox BG. Proc Natl Acad Sci USA. 2008; 105:19194-19198. [PubMed: 19033467] 

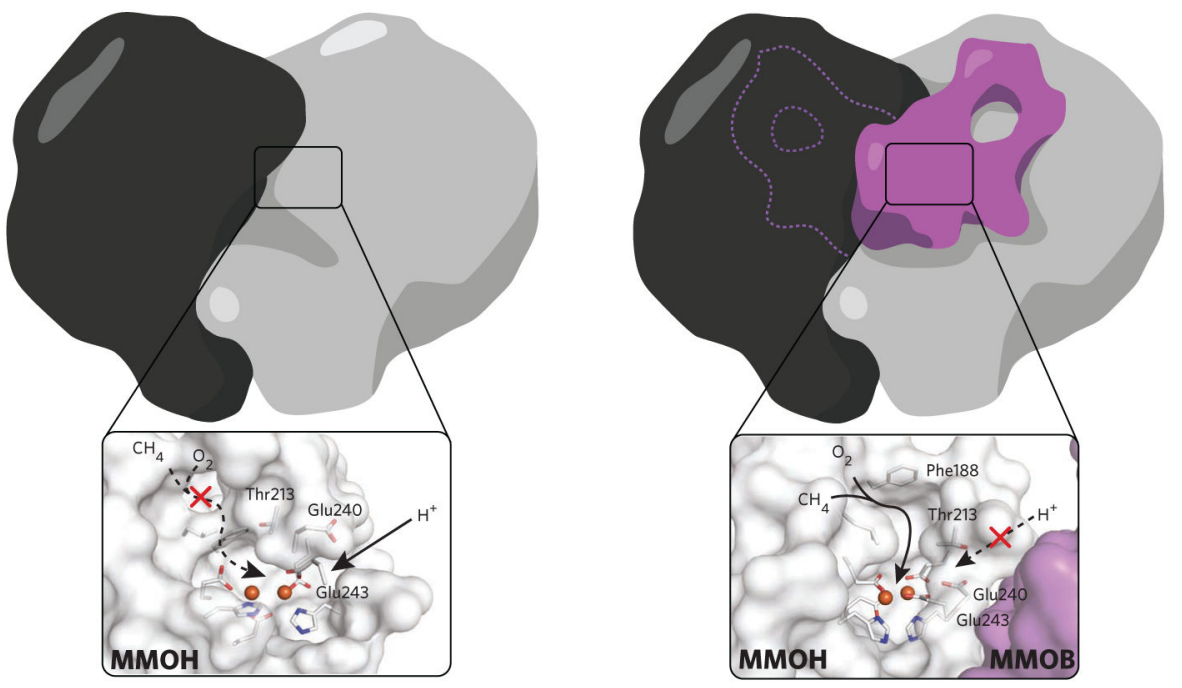

Figure 1.

Protein-protein interactions in the MMOH-MMOB methane oxidation complex modulate access to the active site. Left, MMOH alone (gray and black). Right, complex with MMOB (purple). Iron ions are shown as orange spheres, and key residues are shown as sticks. Dashed arrows indicate a closed pathway, and solid arrows indicate an open pathway. 